\title{
IV. Ein Beitrag zur Kenntnis der isomorphen Vertretung der vier Halogene in Kohlenstoffverbindungen.
}

\author{
Von \\ F. M. Jaeger in Zaandam.
}

(Mit 9 Textfiguren.)

Vor einiger Zeit wurde von B. GoBner ${ }^{1}$ ) eine Abhandlung publiciert über die Krystallformen von Chlorbrom-, Dibrom- und Jodbromnitrophenol, als experimenteller Beitrag zur Kenntnis der isomorphen Vertretung von $C l, B r$ und $J$ in organischen Molekülen. In seiner Zusammenstellung der bisher untersuchten Fälle auf anorganischem und organischem Gebiete geht hervor, daß die directe Isomorphie der genannten Elemente fast in keinem Falle vorliegt, und zweitens, daß die Reihen der organischen Substitutionsproducte dieser Art noch fast nie vollständig untersucht sind.

Ich könnte hinzufügen, daß man sich auch um das Verhalten solcher organischer Derivate in binären geschmolzenen Gemischen noch bis heute gar nicht gekümmert hat.

Vollständig krystallographisch untersucht wurden bis jetzt: p-Chlor-, p-Brom- und p-Jodacetanilid ${ }^{2}$ ), Schmelzpunkt resp. $179^{\circ}, 167,5^{\circ}$ und $181^{\circ} \mathrm{C}$. Die Brom- und Jodverbindungen sind beide monoklin, die Chlorverbindung weicht $a b$ und ist rhombisch.

Obgleich $B r$ - und $J$-Verbindung eine deutliche Analogie der Parameterwerte zeigen, so ist aber die Spaltbarkeit im Vergleiche zu jener der $\mathrm{Cl}$ Verbindung eine durchaus verschiedene ${ }^{3}$. Später zeigte GoBner ${ }^{4}$ ) die

1) Goßner, diese Zeitschr. 1905, 40, 78.

2) Ebenda 1904, 39, 156.

3) Fels, diese Zeitschr. 1900, 32, 386, 406; Müg ge, ebenda 4, 335; Sans oni, ebenda 18, 102; Fels, ebenda 1903, 37, 169; Wilson, ebenda 36, 86 Ref.; Pane bi anco, ebenda 4, 393 .

4) Goßner, diese Zeitschr. 38, 156. 
Dimorphie der drei Verbindungen. Seiner Meinung nach läßt sich hieraus die unregelmäßige Lage der bez. Schmelzpunkte erklären. Dagegen ist die Reihe der drei dihalogensubstituierten Nitrophenole direct isomorph. Nichtsdestoweniger liegen die Schmelzpunlite doch anormal, indem die Jodverbindung diesmal eine abwcichende Stellung einnimmt.

Es sei mir erlaubt, hier den umgekehrten Fall vorzuführen: drei Halogenderivate, deren Schmelzpunkte ganz regelmäßig mit steigendem Atomgewichte um $35_{2}^{10} \mathrm{C}$. steigen, deren Krystallformen aber einer isodimorphen Reihe angehören, bei welcher das Ćhlorderivat die abweichende Stellung einnimmt.

Gleichzeitig werde ich über das Verhalten dicser Verbindungen im Flüssigkeitszustande berichten, weil sich dasselbe als nicht so einfach herausstellte, als ich anfänglich glaubte.

Die Reihe enthält die Methylester der p-Chlor-, p-Brom- und p-Jodbenzoësäure. Üher die beiden ersten Verbindungen habe ich schon früher $\left.{ }^{1}\right)$ Einiges mitgeteilt, was jetzt vervollstïndigt wird. Obendrein sind noch einige Angaben über p-Fluor-, p-Chlor- und p-Jodbenzoësäure hinzugefügt. Den p-Fluorbenzö̈süure-Methylester habe ich dargestellt, aber er ist bei gewöhnlichcr Temperatur eine wasserhelle, farblose, stark anisartig riechende Flüssigkeit, also für krystallographische Bestimmung untauglich.

Die p-Fluor- und p-Jodbenzoësäure habe ich aus p-Fluor-resp. p-Jodtoluol durch Oxydation mittelst Kaliumpermanganat in alkalischer Lüsung hergestellt; das p-Fluortoluol erhielt ich durch die Gefälligkeit des Herrn Prof. Dr. A. F. Holleman in Amsterdam, das p-Jodtoluol stellte ich dar durch Diazotieren von reinstem $p$-Toluidin in Jodkaliumlüsung. Die Säuren wurden aus den Alkalisalzen mittels Salzsäure gefällt und durch mehrmaliges Umkrystallisieren aus Alkohol gereinigt.

Zur Esterification wurden sie in Methylalkohol gelöst und ein Strom trockner Chlorwasserstoflsïure durchgeleitet. Die Ester wurden in der gebräuchlichen Weise gereinigt, durch Schülteln mit Natriumbicarbonatlösung und wiederholtes Umkrystallisieren.

In dieser Weise dargestellt bildet der Fluorbenzo ësäure-Ester eine Flüssigkeit, das Jodderivat aber eine bei $114^{\circ} \mathrm{C}$. schmelzende, farblose Verbindung.

Das Methyl-p-Jodbenzoat, Schmelzpunkt $114^{\circ} \mathrm{C}$., krystallisiert aus Äther + Alkohol in farblosen, schwach anisartig riechenden Nadeln, welche geometrisch sehr rein gebaut sind. Sie haben die Gestalt der Fig. 1.

Rhombisch-bipyramidal.

$$
a: b: c=1,4144: 1: 0,8187 \text {. }
$$

1) J a eger, diese Zeitschr. 1903, 38, 279; N. Jahrb. C. Min. usw. 1903, 1, 1-28. Ausz. diese Zeitschr. 41, 664. 
Beobachtete Formen: a $\{100\}$ vorherrschend, sehr stark glänzend, bisweilen mit feiner, verticaler Streifung rersehen; $p\{210\}$ sehr scharf reflec-

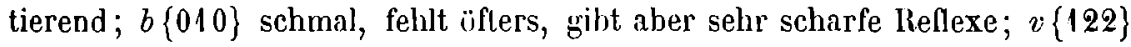
und $q\{011\}$ gut entwickelt; $i\{112\}$ klein und oft günzlich fehlend.

Der Habitus ist nach $\{100\}$ aljgeplatlet, mit Strcckung nach der $c$-Axe.

lig. 1 .

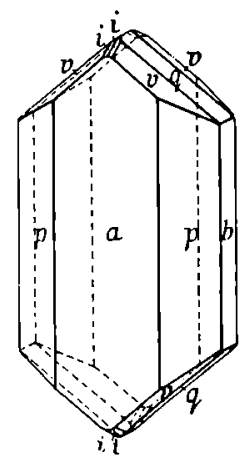

Fig. 2.

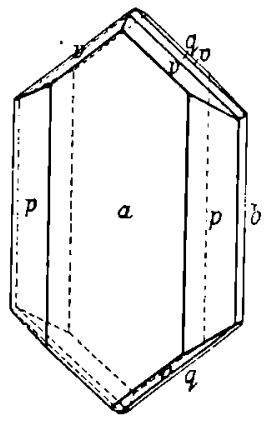

Fig. 3.

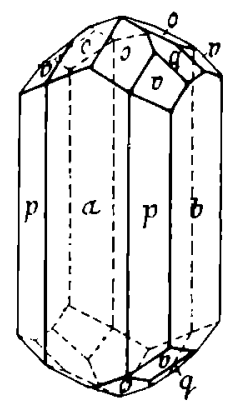

Es wurden gemessen:

$\begin{array}{llc}\text { Gemessen: } & \text { Berechnel: } \\ a: p=(100):(210) & * 35015 \frac{3}{4} & - \\ b: v=(010):(122) & * 5149 & - \\ b: p=(010):(210) & * 5444 \frac{1}{2} & - \\ v: v=(122):(122) & 7623 & 76022{ }^{\prime} \\ b: q=(010):(011) & 5024 ! & 5041 \frac{1}{2} \\ a: v=(100):(122) & 7729 & 7723 \\ v: v=(122):(122) & 2542 & 2541 \\ q: q=(011):(011) & 7912 & 7911 \\ v: q=(122):(011) & 12501 & 1237 \\ p: q=(210):(011) & 6823 & 6833 \\ v: i=(122):(112) & 1701 . & 1643 \frac{1}{3} \\ i: i=(112):(112) & 433 & 4255 \frac{1}{3}\end{array}$

Spaltbar nach $\{010\}$.

Die optische $\Lambda x$ enebene ist $\{001\}$ mit der $b$-Axe als erster Mittellinie. Der scheinbare Axenwinkel in $\alpha$-Monobromnaphtalin ist ca. $80^{\circ}$; die Dispersion ist $\varrho<v$. Auf $a, p$ und $b$ parallele Auslöschung.

Das specifische Gewicht der Krystalle beträgt 2,020 bei $10^{\circ} \mathrm{C}$.; das $\ddot{\Lambda}$ quivalentvolum ist mithin $=129, \% 0$.

Topische Axen: $\%: \psi: \omega=6,8179: 4,8203: 3,9464$.

Aus den oben angeführten Bestimmungen geht die directe Isomorphie der Brom- und Jodverbindungen zweifellos hervor. Zum Vergleich habe ich hier die bezüglichen Daten für beide nochmals neben einander gestellt: 
Ein Beitr. z. Kenntn. der isom. Vertret. der vier Halogene in Kohlenstoffverbind. 19

p-Brombenzoësäure-Ester

(Figg. 2 u. 3).

Rhombisch-bipyramidal.

$a: b: c=1,3967: 1: 0,8402$.

Formen: $\{100\},\{010\},\left\{\begin{array}{lll}0 & 1\end{array}\right\},\{210\}$, $\{112\},\{122\}$.

Aul $\{100\}$ feine Streifung parallel der $c-\Lambda x e$.

Spaltbar undeutlich nach $b$.

Optische Axenebene $\{001\}$; erste Mittellinie die $b$-Axe.

Winkelwerte:

$$
\begin{aligned}
& a: p=35^{0} 16^{\prime} \\
& b: v=5149 \\
& o: o=4255
\end{aligned}
$$

usw.
p-Jodbenzoësäure-Ester.

Rhombisch-bipyramidal.

$$
a: b: c=1,4144: 1: 0,8187 \text {. }
$$

Formen: $\{100\},\{010\},\{011\},\{210\}$, $\{112\},\{122\}$.

$\Lambda u f\{100\}$ feine Streifung parallel der $c$-Axe.

Spaltbar nach $b$.

Oplische Axenebene $\{001\}$; erste Mittellinie die $b$-Axe.

Winkelwerte:

$$
\begin{aligned}
& a: p=34^{\circ} 56^{\prime} \\
& b: v=5110 \\
& o: o=4350
\end{aligned}
$$

usw.

Die scheinbaren Axenwinkel beider Verbindungen sind gleich, wenn man die Jodverbindung in a-Monobromnaphtalin, das Bromderivat in Cassiaül beobachtet; die Dispersion in beiden Fällen ist ron entgegengesetztem Charakter.

Deshalb sind es hier $B r$ - und $J$-Verbindung, welche sich analog verhalten; dagegen zeigt die Chlorverbindung das abweichende Verhalten. Die Schmelzpunkte hingegen steigen ganz regelmäßig: $\$ 4.0 \mathrm{C}$., $792^{10}, 114^{\circ} \mathrm{C}$.

Ich habe dann das Verhalten dieser Körper im Flüssigkeitszustande erforscht durch Bestimmung der binären Schmelzcurven, welche in der Fig. 4 wiedergegeben sind. Die Concentration ist in Molekülprocenten ausgedrückt.

Die Schmelzcurve der $B r-J-V e r-$ bindung weicht praktisch nicht

Fig. 4.

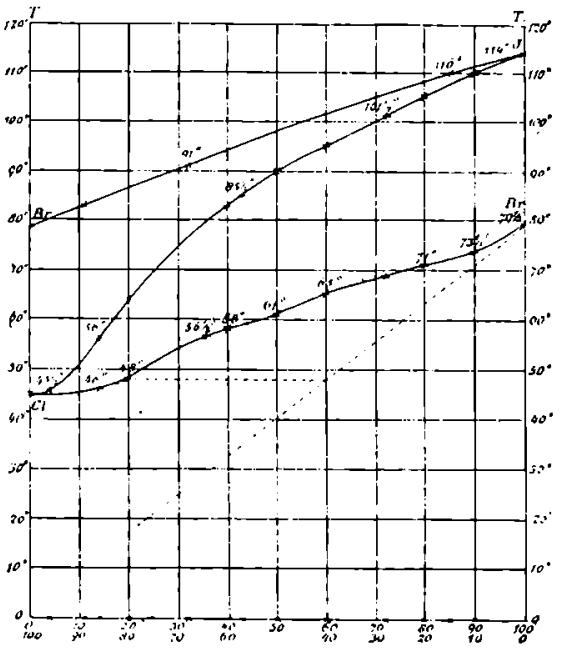
von ciner geraden Linie $a b$; die Differenzen sind nur geringfügig. Die Schmelzpunktserniedrigung des Jodderivates ist deshalb praktisch direct proportional der Anzahl hinzugefügter Moleküle der Bromverbindung.

Die Schmelzcurven der $C l-J$ - und $C l-B r$-Verbindung haben analoge Form. Alle beobachteten Endschmelzpunkte liegen hier zwischen niedrigstem und 
höchstem Schmelzpunkt. Beide Schmelzcurven gehören zum steigenden Typus Roozebooms. Die unteren Ïste sind thermometrisch nicht bestimmbar, obgleich die $A$ nwesenheit derselben bewiesen wurde, und zwar, daß sie in relativ großer Entfernung liegen vom oberen Aste, wenigstens an der Seite der höchstschmelzenden Verbindung. Die Ursache der unscharfen Beslimmung dieses Astes ist gelegen in dem sehr flachen Verlaufe der Abkühlungscurve, so daß eine bestimmte Lage für den Punkt, der den unstetig stattfindenden Wärmeeffect während des Abkühlens angeben soll, nicht anzugeben ist.

Die Bestimmung der Mischungsgrenzen durch Untersuchung der festen Phasen, welche mit Lüsungen von bekannter Concentration im Gleichgewicht sind, schlägt fehl infolge von Schwierigkeiten analytischer Art. Es wurde deshalb versucht, dic Mischungsgrenzen nach krystallographischer Methode zu bestimmen. Es wurden dazu Lösungen von Gemischen der beiden

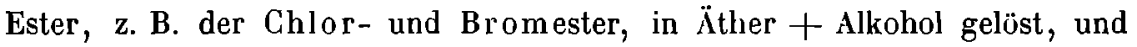
die durch langsame Verdampfung erhaltenen Mischkrystalle erst gemessen und dann ihre Zusammensetzung ermittelt durch den Endschmelzpunkt. Denn wenn man annehmen darf, daß bei dieser Weise des Operierens das letzte feste Teilchen wirklich in stabile $m$ Gleichgewicht mit der umgebenden flüssigen Masse ist, dann geben die so gefundenen Endschmelzpunkte durch Vergleich mit dem schon gefundenen oberen Aste der Schmelzcurve auch wirklich die molekularprocentische Zusammensetzung der Mischkrystalle an.

Nimmt man wenig $C l$-Ester und viel $B r$-Ester, dann krystallisieren aus der alkoholischen Lösung monokline Mischkrystalle, welche genau die Gestalt des monollinen Cl-Esters besitzen. Von solchen Krystallen wurden nie Schmelzpunkte beobachtet, welche höher als $46,5^{\circ} \mathrm{C}$. waren. Beim umgekehrten Verhältnis der Componenten dagegen setzen sich Mischkrystalle vom Typus des rhombischen $B r$-Esters ab. An diesen Krystallen beobachtete ich nun merkwürdigerweise Schmelzpunkte von $79,5^{\circ} \mathrm{G}$. bis zu $4.7^{\prime \prime} \mathrm{C}$., niedriger als $47^{\circ} \mathrm{C}$. fand ich dieselben nie.

Es scheint daher - unter Annahme, daß die Endglieder der monoklinen Reihe praktisch nicht von Krystallen differieren, welche den Schmelzpunkt $461^{10}$ oder $47^{\circ} \mathrm{C}$. aufweisen $-\mathrm{da \beta}$ rhombische Mischungsphasen existieren können, welche sich beim »Übergangspunkte $4.7^{\circ}$ unmittel bar den monoklinen Phasen anschließen!

Nun habe ich aber gefunden, daß rhombische Mischkrystalle von sehr verschiedenen Schmelzpunkten, zusammen im geschlossenen Bohre während mehrerer Monate im Dunkeln aufbewahrt, matt und zum Teil undurchsichtig werden, mit rauher Oberfläche, sobald ihr Schmelzpunkt unter$\mathrm{h}$ alb $6 \ddot{3}^{\circ} \mathrm{C}$. fällt $\mathrm{Es}$ ist obendrein hervorzuheben, daß die rhombischen Mischungen unterhalb $6: 0^{0}$ um so schlechter ausgebildet und krummflächig, mehr und mehr tordiert, oft als in einem Zwangzustande befind- 
Ein Beitr. z. Kenntn. der isom. Vertret. der vier Halogene in Kohlenstoffverbind. 21

lich, erscheinen, je mehr ihr Schmelzpunkt von $60^{\circ} \mathrm{C}$. abweicht und sich $47^{\circ} \mathrm{C}$. nähert.

Ich muß deshalb schließen, daß, unter Annahme der oben herbeigeführten Gleichgewichtshypothese, alle rhombischen Mischungen unterhalb $66^{\circ} \mathrm{C}$. schmelzend, metastabile Zustände vorstellen, welche sich mit großer Langsamkeit im festen Zustande spalten, um sich partiell in monokline Glieder der Reihe umzusetzen.

Die Schmelzcurve $i, c$ bekommt dann die Gestalt der Fig. 4; die erwähnten metastabilen Zustïnde liegen alle auf dem punktierten Teile des unteren Astes rechts, welcher Ast die Zusammensetzung der mit der geschmolzenen Masse coëxistierenden Mischkrystalle vorstellt. Die »stabile « Lücke in der Mischungsreihe erstreckt sich deshalb dann von circa $18 \%$ bis $\mathrm{zu} 60 \%$ des Bromesters.

Aus dem Vorigen läßt sich folgern, daß durch die sehr langsame Umwandlung der rhombischen Mischungen mit Mischungsverhältnissen unterhalb $60 \%$ des Bromesters, in monoklinen Mischungen mit weniger als ( $0 \%$ des Esters, neben rhombischen, auch in dieser Weise keine scharfe Bestimmung der Mischungsgrenzen zu erbringen ist.

Noch schwieriger wie im beschriebenen Falle ist dies im System des $\mathrm{Cl}$ - und $J$-Esters. Dort ist das Endglied der monoklinen Reihe noch viel nïher an der Coordinatenaxe, als im obigen Falle. Infolge des sehr großen Unterschiedes der Löslichkeit beider Verbindungen bekommt man hier aus alkoholischen Lüsungen nie etwas anderes, als rhombische sehr feine Nädelchen, während die monoklinen Phasen so undeutlich krystallisieren, daß sie für genauere Untersuchung gänzlich ungeeignet sind.

Dagegen krystallisieren die $B r$ - und $J$-Verbindungen leicht in allen Verhältnissen. Die Winkelwerte der schünen, ganz klaren und von optischen $\Lambda$ nomalien freien Mischkrystalle differiren nur wenig von jenen der zwei Componenten. Sie besitzen analoge, molekulare Structur, und auch ihre $\ddot{\Lambda}_{\text {quivalentvolumina differieren nur unerheblich im Vergleich mit dem }}$ Volum des festen $\mathrm{Cl}$-Esters.

In bezug auf die Schmelzpunktserniedrigung der höchstschmelzenden Verbindung durch Zusatz der niedrigerschmelzenden ist noch hervorzuheben, daß diese nicht - wie im System $B r$ - und $J$-Ester - proportional der Anzahl zugesetzter Moleküle ist. (Unrichtige »Küstersche Regel«). Bei den Mischungen des Cl- und $J$-Esters liegen diese Erniedrigungen stets auf einer Curve, welche oberhalb der Geraden der proportionellen Schmelzpunktserniedrigung liegt; im System $\mathrm{Cl}$ - und $\mathrm{Br}$-Ester aber auf einer zweiperiodischen Curve, welche unterhalb der genannten geraden Linie gelegen ist.

Schließlich sei bemerkt, daß die sich aus alkoholischen Lösungen absetzenden Mischkrystalle einen größeren Gehalt am höher schmelzenden 
Componenten besitzen als die Lösung, aus welcher sie sich abgesetzt haben. So kamen aus einer Lösung, welche $20 \% B r$-Ester und $80 \% C l$-Ester enthält, erst rhombische Mischkrystalle, welche einen Schmelzpunkt von $57^{\circ} \mathrm{C}$. aufwiesen, correspondierend mit ciner beträchllich höheren Procentzahl des Bromesters als $20 \%$.

Fig. :3.

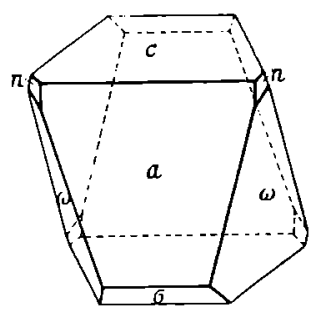

Das Chlorderivat, welches monoklin ist, mit $a: b: c=1,8626: 1: 3,4260$ und $\beta=64{ }^{\circ} 11^{\prime}$, und mit den Formen: $a\{100\}, c\{001\}, \sigma\{\overline{1} 02\}$, $x\{210\}, q\{011\}, c(T 11\}, o\{111\}$ und $\xi\{113\}$, zeigt einen Habitus, welcher in keinem Falle jenem der anderen zwei Derivate nahe kommt, obgleich dieser Habitus, wie die Figuren 5-9 zeigen, in hohem Grade veränderlich ist mit der Wahl des Lösungsmittels und der Temperatur während der Krystallisation.

Fig. 6.

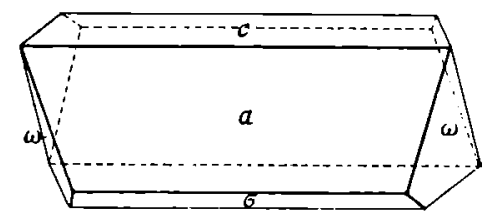

Fig. 8.

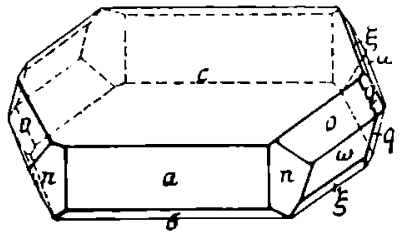

Fig. 7 .

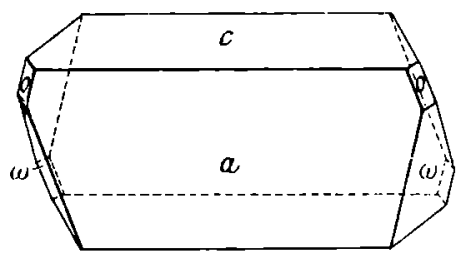

Fig. 9.

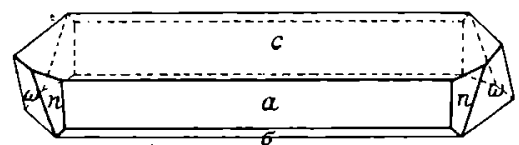

Der Habitus der $B r$ - und $J$-Verbindungen dagegen ist vollkommen analog; beim $J$-Ester ist er obendrein unter sehr veränderten Umständen der Krystallisation ziemlich constant (Fig. 8), während er beim Br-Derivate noch einigermaßen veränderlich erscheint, wie die Fig. 8 und 9 zeigen. Mit steigendem Atomgewichte nimmt daher hier die Tendenz zur Variation des Habitus unter variierten Umständen während des Krystallisierens regelmäßig ab.

Das specifische Gewicht und die topischen Parameter sind:

Cl-Ester: $\quad d_{20^{0}}=1,382 ; V=123,37 ; \chi: \psi: \omega=5,1731: 2,7771: 9,5153$. $B r$-Ester: $d_{20^{0}}=1,689 ; V=127,29 ; \chi: \psi: \omega=6,6611: 4,7691: 4,0070$. J-Ester: $\quad d_{200}=2,020 ; \nabla=129,70 ; \chi: \psi: \omega=6,8179: 4,8203: 3,9464$. 
Alle diese Tatsachen gestatten keine andere Erklärung, als die Annahme, daß die drei halogenierten Benzoësüureester dimorph sind, und zwar muß der $C l$-Ester noch eine labilere rhombische, die $B r$ - und $J$-Verbindungen aber noch eine labilere monokline Form besitzen.

In einer Abhandlung von Bruni und Padoa ${ }^{1}$ ) wird von Helene Duse ein wonokliner p-Brombenzoësäure-Methylester beschrieben, und zwar um eine „Isomorphie zu beweisen mit dem analogen p-Nitrobenzoësäure-Ester. Die angeführten Messungen aber stehen in gar keinem Zusammenhange mit denjenigen, welche am Chlorderivate ausgeführt worden sind, so daß diese »monokline " Form jedenfalls nicht die gesuchte sein kann. Die Daten und Winkelwerte, welche dort angeführt werden, stimmen sogar in keiner Ilinsicht mit den Werten, welche ich am Bromderivate fand, während doch die beobachtete Krystallform als durchwegs auftretende, daher stabile Form des Bromesters beschrieben wird. Es kommt mir zweifelhaft vor, ob diese Messungen der Italienerin wohl richtig sind; vielleicht aber hat sie gar keinen p-BrombenzoësäureEster in Händen gehabt.

Alle Versuche, die genannte »monokline* Form des Bromderivates zu bekommen, sind bisher ohne jedes Resultat gewesen.

Um eine eventuell vorliegende Dimorphie dieser Körper zu prüfen, benutzte ich die Lehmannsche mikroskopische Methode, und zwar mittels des von ihm construierten Krystallisationsmikroskopes.

In keinem Falle aber konnte ein positives Resultat erzielt werden. Wohl beobachtete ich bei diesen Körpern anscheinend zwei verschiedene Arten der Krystallisation, namentlich einerseits lange, feine, andererseits parallelogrammatische, sehr flache Nadeln. Beide Arten zeigten aber parallele Auslöschung und unterschieden sich überhaupt nur " so unbeträchtlich, daß, mit Rücksicht auf die Tendenz zur Variation des Habitus, welche zweien dieser Körper so sehr eigen ist, ich es nicht wage, die beiden Krystallarten als "verschieden ", im Sinne einer vorliegenden Dimorphie, zu erklären. Auch Mischungen der Ester verhielten sich so.

Obgleich ich deshalb eine Isodimorphie hier sehr wahrscheinlich erachte, so ist dieselbe keineswegs streng bewiesen, und ebenso wenig, ob es sich hier um enantiotrope oder monotrope Modificationen handelt.

Schließlich führe ich noch einige Daten an bezüglich der vier halogenierten Benzoësäuren.

1. Die p-Chlorbenzoësäure, Schmelzpunkt $243^{\circ} \mathrm{C}$., ist von $\mathrm{Fels}^{2}$ ) gemessen. Sie ist monoklin, mit: $a: b: c=1,2738: 1: 3,3297$ und $\beta=$

1) Bruni e Padoa, Gazz. chim. ital. 1904, 34a 133 ; Rendic. Lincei 1903, 5a, 12, 348. S. das Ref. am Schlusse dieses Hefles S. 76.

2) Fels, diese Zeitschr. $1900,32,389$. 
24 F. M. Jaeger. Ein Beitr. z. Kenntn. der isom. Vertret. der vier Halogene usw.

$78^{0} 244^{\prime}$, wenn die beobachteten Formen als $\{100\},\{001\},\{\overline{2} 07\},\{111\}$, $\{\overline{2} 33\},\{322\},\{\overline{4} 11\}$ angenommen werden.

2. Die p-Fluorbenzoësäure, Schmelzpunkt $182^{\circ} \mathrm{C}$., ist gleichfalls monoklin. Legt man den auftretenden Flächen die Symbole $\{100\},\{001\}$, $\{\overline{203}\},\{\bar{r} 03\}$ und $\{043\}$ bei, so gestaltet sich das Parameterverhältnis zu $a: b: c=1,192: 1: 3,183$ und $\beta=78^{\circ} 16^{\prime}$; die Messungen können nur als annähernde gelten.

Der Schmelzpunkt der p-Fluorsäure wird durch Zusatz der Cl-Verbindung erhöht.

Einige Daten: $(00 \bar{T}):(20 \overline{3})=69^{0} 56^{\prime} ;(\overline{2} 03):(\overline{1} 03)=15^{0} 36^{\prime} ;(\overline{1} 03):$ $(100)=16^{0} 14^{\prime} ;(001):(100)=78^{0} 16^{\prime} ;(001):(043)=76^{0} 28^{\prime} ;(043):$ $(04 \overline{3})=2704^{\prime}$.

Der Habitus ist dünntaflig nach $c$, mit rechteckigem Umriß. Krystalle aus Äther + Alkohol. Auf $c$ parallel orientierte Auslöschung.

3. Die p-Brombenzoësäure, Schmelzpunkt $252^{\circ} \mathrm{C}$., wurde in sehr kleinen, mangelhaften Krystallen aus Benzol + Äthylacetat erhalten. Sie sind monoklin und wahrscheinlich isomorph mit den beiden anderen Säuren. Der Winkel $\beta$ beträgt circa $78 \frac{20}{3}$.

4. Die p-Jodbenzoësäure, Schmelzpunkt $267^{\circ} \mathrm{C}$., konnte bisher nicht in geeigneter Form erhalten werden ihrer geringen Löslichkeit wegen.

Die Schmelzpunkte der vier Verbindungen liegen hier ganz regelmäßig. 